\title{
Notas nomenclaturais sobre espécies brasileiras de Baccharis (Asteraceae) ${ }^{1}$
}

\author{
Nomenclatural notes on Brazilian species of Baccharis (Asteraceae)
}

\author{
Gustavo Heiden $^{2}$, José Fernando A. Baumgratz ${ }^{3}$ \& Roberto Lourenço Esteves ${ }^{4}$
}

\begin{abstract}
Resumo
Com base em estudos taxonômicos recentes em Baccharis é restabelecido o nome $B$. montana DC., novos sinônimos são propostos para B. lateralis Baker, B. montana DC., B. oblongifolia (Ruiz \& Pav.) Pers., B. parvidentata Teodoro, B. retusa DC., B. rufidula (Spreng.) Joch.Müll., B. tridentata Vahl e B. trinervis (Lam.) Pers., e lectótipos são designados para B. elaeagnoides Sch.Bip. ex Baker e B. schultzii Baker.

Palavras-chave: Astereae, Compositae, taxonomia.
\end{abstract}

\begin{abstract}
Based on recent taxonomic studies on Baccharis, the name B. montana DC. is reinstated, new synonyms are proposed for B. lateralis Baker, B. montana DC., B. oblongifolia (Ruiz \& Pav.) Pers., B. parvidentata Teodoro, B. retusa DC., B. rufidula (Spreng.) Joch.Müll., B. tridentata Vahl, and B. trinervis (Lam.) Pers., and lectotypes are designated for B. elaeagnoides Sch.Bip. ex Baker and B. schultzii Baker.
\end{abstract}

Key words: Astereae, Compositae, taxonomy.

\section{Introdução}

Baccharis L. compreende ca. 360 (Nesom \& Robinson 2006) a 500 espécies (Malagarriga Heras 1976), distribuídas originalmente nas Américas, desde o sul do Canadá até o sul da América do Sul (Fielding 2001; Giuliano 2001). Os principais centros de diversidade do gênero são os Andes, desde a Colômbia até a região central do Chile e da Argentina, e as regiões montanhosas do sudeste do Brasil, do Uruguai e do leste do Paraguai. O indumento em diminutos tufos de tricomas nos caules e folhas, com os tricomas possuindo uma única célula basal, e as flores unissexuais geralmente em espécimes distintos, têm sido considerados prováveis sinapomorfias do gênero (Müller 2006).

Baccharis está representado no Brasil por ca. 150 espécies, com maior diversidade nos campos rupestres e formações savânicas no Domínio do Cerrado, nos campos de altitude no Domínio da Mata Atlântica e nos campos de clima temperado no Domínio do Pampa.

Com base em estudos taxonômicos recentes em Baccharis (Heiden 2009), é proposto o restabelecimento de um binômio, o reconhecimento de novos sinônimos para oito espécies e a proposição de duas lectotipificações.

\section{Material e Métodos}

As proposições são baseadas em revisão bibliográfica, análise de coleções históricas e recentes, incluindo tipos e fotos de tipos, disponíveis nos herbários B, C, CEPEC, F, FCAB, G, GUA, HAS, HB, HBG, HPNI, HRJ, HUEFS, HURG, IAC, ICN, IPA, JPB, K, M, MBML, MG, MO, NY, P, PACA, PEL, R, RB, RBR, RFFP, RUSU, S, SMDB, SP, UFP, UFRN, US e $\mathrm{W}$, e em observações de campo realizadas nos principais centros de diversidade do gênero no Brasil.

\section{Resultados e Discussão}

Baccharis lateralis Baker in Martius, Eichler \& Urban, Fl. bras. 6(3): 100. 1882. Tipo: BRASIL, , $F$. Sellow 1146 (síntipo $\mathrm{B} \dagger$, foto em F!).

Baccharis schultzii Baker in Martius, Eichler \&Urban, Fl. bras. 6(3): 78. 1882. Tipo:BRASIL. MINAS GERAIS: Poços de Caldas, 2.II.1868, ơ, A.F. Regnell III-758 (lectótipo aqui designado R!), syn. nov.

\footnotetext{
'Parte da dissertação de Mestrado do primeiro autor, desenvolvida na Escola Nacional de Botânica Tropical, Instituto de Pesquisas Jardim Botânico do Rio de Janeiro. ${ }^{2}$ Universidade de São Paulo, Depto. Botânica, Instituto de Biociências, R. do Matão, Tv. 14, 321, São Paulo, SP, 05508-900, Brasil. gustavo.heiden@gmail.com ${ }^{3}$ Instituto de Pesquisas Jardim Botânico do Rio de Janeiro, Diretoria de Pesquisa Científica, R. Pacheco Leão 915, Rio de Janeiro, RJ, 22460-030, Brasil. jbaumgra@jbrj.gov.br ${ }^{4}$ Universidade do Estado do Rio de Janeiro, IBRAG, Depto. Biologia Vegetal, R. São Francisco Xavier 524, Rio de Janeiro, RJ, 20550-900, Brasil. esteves.vr@gmail.com
} 
Baker (1882) classificou Baccharis schultzii na série Oblongifoliae e B. lateralisna série Cuneifoliae. Embora posicionadas em séries taxonômicas distintas, ambas as espécies foram caracterizadas pelo autor por apresentar capítulos aglomerados em racemos axilares, subtendidos por folhas de maior comprimento que o eixo da capitulescência. $\mathrm{O}$ exame da foto do síntipo de B. lateralis, do lectótipo de B. schultzii e de vários espécimes permitiu concluir que ambos os nomes se referem ao mesmo táxon. Como os dois binômios foram publicados pelo mesmo autor na mesma obra, optou-se por aceitar o nome B. lateralis, pois salienta um caráter diagnóstico do táxon - os racemos de capítulos axilares dispostos ao longo dos ramos. Além disso, após o exame dos síntipos (Riedel 538-540; Regnell III-758; Widgren 257) de B. schultzii, o espécime Regnell III-758(R) é designado como lectótipo por apresentar ramos completos, além de capítulos femininos, flores e cipselas em bom estado de conservação.

Baccharis montana DC., Prodr. 5: 404. 1836. Tipo: BRASIL. RIO DE JANEIRO: O’, P. Lund 22 (síntipo G-DC, foto!); Serra da Estrela, O", P. Lund 106 (síntipo G-DC, foto!).

Baccharis elaeagnoides Steud. ex Baker in Martius, Eichler \& Urban, Fl. bras. 6(3): 53. 1882. Baccharis semiserrata var. elaeagnoides (Steudel ex Baker) G.M.Barroso, Rodriguésia 28(40): 118. 1976. Baccharis semiserrata var. elaeagnoides (Steudel ex Baker) Govaerts, World Checkl. Seed Pl. 2(1): 9. 1996. Tipo: BRASIL. SÃO PAULO: Jundiaí, I.1834, o, P. Lund s.n. (lectótipo aqui designado $\mathrm{C}$ !), syn. nov.

O exame do protólogo e de imagens dos síntipos de Baccharis montana demonstra que o material tipo corresponde à mesma espécie que Steudel identificou in schedulae como B. elaeagnoides e que Baker (1882) utilizou para descrever posteriormente essa espécie. Conseqüentemente, $B$. montana é o nome correto e mais antigo para o táxon comumente referido como $B$. elaeagnoides ou $B$. semiserrata var. elaeagnoides no Brasil, motivo pelo qual se propõe o novo sinônimo. A lectotipificação de $B$. montana não é feita no presente estudo, pois as imagens dos tipos não permitem apontar qual espécime possui capítulos em melhor estado de conservação. Baker (1882), ao descrever $B$. elaeagnoides, listou vários espécimes (Lund s.n.; Glaziou 540; Glaziou 1050; Regnell I-246; Warming 220). O material coletado por Lund s.n. (C) foi aqui designado como lectótipo por se constituir de um ramo completo, com folhas, capitulescências e capítulos femininos portando flores e cipselas.
Baccharis oblongifolia (Ruiz \& Pav.) Pers., Syn. Pl. 2(2): 424. 1807. Molina oblongifolia Ruiz \& Pav., Syst. veg. fl. peruv. chil. p. 203. 1798. Tipo: in Regni Chilensis silvis, versus Conceptionis urbem, et Hualpen tractus [conforme Müller (2006), provavelmente centro do Peru], ᄋ, H. Ruiz \& J. Pavón s.n. (holótipo MA; isótipos B-fragmentos, G 169377 foto!).

Baccharis brachylaenoides DC., Prodr. 5: 421. 1836. Psila brachylaenoides (DC.) Aristeg., Fl. Venez. 10(1): 316. 1964. Tipo: BRASIL. RIO DE JANEIRO: Corcovado, ○ \& O’, P. Lund 609 (holótipo G-DC, foto!).

Baccharis venulosa DC., Prodr. 5: 421. 1836. Tipo: in Peruviae montibus Oronocensibus, ㅇ \& o', T.X.P. Haenke s.n. (holótipo G-DC 200395, foto!; isótipo $\mathrm{B} \dagger$, foto $\mathrm{F}$ !).

Baccharis maguireana Malag., Mem. Soc. Ci. Nat. La Salle 37(107): 138. 1976. Tipo: VENEZUELA. AMAZONAS: Serranía Yutaje, Río Manapiare, in small savanna along left fork, Cano Ytaje, $1250 \mathrm{~m}$, 12.II.1953, o, B. Maguire et C.K. Maguire 35179 (holótipo P, foto!; isótipo NY, foto!), syn. nov.

Baccharis oblongifolia possui ampla distribuição geográfica na América do Sul (Argentina, Bolívia, Brasil, Colômbia, Equador e Peru). Espécimes coletados em encostas e áreas de baixa altitude adjacentes ao Escudo das Guianas apresentam folhas com um padrão de nervação reticulado mais proeminente. Esta característica foi utilizada para circunscrever $B$. venulosa (Candolle 1836) e $B$. maguireana (Malagarriga Heras 1976), ambas citadas para o Brasil. A distribuição geográfica de espécimes com essa característica se sobrepõe completamente com a área de ocorrência dos indivíduos típicos de $B$. oblongifolia, tendo sido essa característica foliar considerada apenas uma variação morfológica intraespecífica (Heiden 2009), sem valor diagnóstico para o estabelecimento de variedades ou espécies autônomas. Além disso, características vegetativas e reprodutivas presentes nos espécimes com nervação reticulada proeminente são comuns também em espécimes de B. oblongifolia (Heiden 2009), razão pela qual esses binômios são propostos como sinônimos.

Baccharis parvidentata Teodoro, Mem. Soc. Ci. Nat. 37: 138. 1976. Tipo: BRASIL. RIODE JANEIRO: Itatiaia, altitude ca. $2000 \mathrm{~m}$ nos campos da Serra do Itatiaia, 1.I.1896, o, E. Ule 641 (holótipo R!).

Baccharis nassauvioides Oliveira-Deble \& Deble, Bonplandia 17(1): 20. 2008. Tipo: BRASIL. RIO DE JANEIRO: Itatiaia, Prateleiras, $2200 \mathrm{~m}$, 8.V.1975, o, A.M. Camerik 57a (holótipo RB!; isótipo RUSU!), syn. nov. 
Oliveira-Deble \& Deble (2008) descrevem Baccharis nassauvioides com base em espécimes coletados em Itatiaia e a circunscrevem com base em características das folhas, capítulos e flores, diferenciando-a de B. arassatubaensis Teodoro (endêmica da Serra de Araçatuba, na cadeia da Serra do Mar, divisa entre Paraná e Santa Catarina) e B. serrula Sch.Bip. ex Baker (endêmica da Serra da Lapa, no Parque Nacional da Serra do Cipó, Minas Gerais), sem entretanto relacioná-la com $B$. parvidentata, com a qual seria simpátrica. O estudo dos respectivos exemplarestipo demonstra que as descrições de ambas as espécies se sobrepõem e não há diferenças que permitam distinguir $B$. nassauvioides de B. parvidentata, razão pela qual se propõe o novo sinônimo.

Baccharis retusa DC., Prodr. 5: 412. 1836. Tipo: BRASIL. SÃO PAULO: O', F. Sellow s.n. [M.I.B. 479] (holótipo G-DC, foto!).

Baccharis salzmannii DC., Prodr. 5: 409. 1836. Tipo: BRASIL. BAHIA: 1831, O", P. Salzmann s.n. (holótipo G-DC; isótipos G 169373 fotos!;HBG;K53031 foto!, K 53032 foto!; P-3ex), syn. nov.

Baccharis halimimorpha DC., Prodr. 5: 411. 1836. Tipo: BRASIL. BAHIA: IV.1831, O',J. Lhotsky s.n. (holótipo G-DC 200467, foto!), syn. nov.

Baccharis ramosissima Gardner, London J. Bot. 7: 84. 1848. Tipo: BRASIL. MINAS GERAIS: pr. Formigas, VII.1840, O’, G. Gardner 4912 (holótipo $\mathrm{BM}$; isótipo $\mathrm{B} \dagger$, foto em $\mathrm{F}$ !), syn. nov.

Barroso (1976) tratou Baccharis ramosissima, que havia sido sinonimizada por Baker(1882) a B. retusa, e B. salzmannii (incluindo B. halimimorpha) como espécies autônomas, diferenciando-as pelo tamanho, forma e dentição das margens das folhas, características essas que se sobrepõem na chave e nas descrições. Com base em tipos e fotos dos tipos e exame de coleções de herbários provenientes de toda a área de ocorrência de $B$. retusa (desde a Bahia até o Uruguai), foi observado que nas folhas, o tamanho, a forma e a dentição das margens são muito variáveis. Devido à ausência de características distintivas nos capítulos e flores e à variabilidade morfológica das características foliares e das capitulescências, admite-se uma circunscrição ampla para $B$. retusa, não sendo possível reconhecer esses três táxons como espécies autônomas, razão pela qual se propõe $B$. halimimorpha, B. ramosissima e $B$. salzmannii como sinônimos.

Baccharis rufidula (Spreng.) Joch.Müll., Syst. Bot. Monogr. 76: 306. 2006. Conyza rufidula Spreng., Neue Entd. 2: 141. 1821. Tipo: BRASIL: Otto s.n. [ex Herb. Sch.Bip.] (holótipo P, foto!).
Baccharis vernonioides DC., Prodr. 5: 422. 1836. Tipo: BRASIL. MINAS GERAIS:Mariana, $M$. Vauthier 268 (holótipo G-DC 105568, foto!; isótipo G-DC 136724, foto!).

Baccharis inamoena Gardner, London J. Bot. 4: 81. 1848. Pseudobaccharis inamoena (Gardner) Teodoro, Contrib. Inst. Geobiol. 2: 47. 1952. Tipo: BRASIL. MINAS GERAIS: Morro Velho, IX.1840, G. Gardner 4918 (holótipo BM; isótipos G; P, foto!; $\mathrm{R}$ !, W), syn. nov.

Müller (2006) reconheceu a prioridade de Conyza rufidula (equivocadamente citada como "Vernonia rufidula Spreng.") sobre Baccharis vernonioides e apropriadamente propôs a combinação nova B. rufidula (Spreng.) Joch.Müll. No mesmo ano, Oliveira et al. (2006) consideraram equivocadamente B. rufidula Sch.Bip. (in schedulae) como tendo prioridade sobre a nova combinação e propuseram o restabelecimento de $B$. vernonioides.

O estudo de isótipos de Baccharis inamoena, binômio não referido na revisão de Barroso (1976), permitiu reconhecer esse táxon também como um sinônimo de B. rufidula.

Baccharis tridentata Vahl, Symb. bot. 3:98. 1794. Tipo: URUGUAI. MONTEVIDEO: Q̨e Ơ, P. Commerson s.n. (holótipoP-218308, foto!; isótipos C22498!; P-Lam, foto!). Baccharis illinita DC., Prodr. 5: 412. 1836. Tipo: BRASIL.SÃO PAULO: @, F. Sellow s.n. [M.I.B. 502] (holótipo G-DC, foto!; isótipo R!), syn. nov.

Baccharis tridentata se caracteriza pelo hábito subarbustivo xilopodífero, folhas coriáceas $(1,5-6 \times$ 0,8-3,7 cm), com 3 nervuras acródromas basais, panículas terminais e capítulos com invólucro campanulado. Barroso (1976) tratou Baccharis illinita como uma espécie autônoma e distinta de $B$. tridentata, diferenciando-a apenas pelo formato e cerosidade das folhas. O exame do isótipo de $B$. illinita (R), representado por um ramo com folhas e portando inúmeros capítulos femininos íntegros e com flores em bom estado de preservação, é congruente com a descrição original e demonstra que o tipo desse binônio representa um espécime de $B$. tridentata, justificando a proposta de sinonimização.

Baccharis trinervis (Lam.) Pers., Syn. pl. 2: 423. 1807. Conyza trinervis Lam., Encycl. 2: 85. 1786. Heterothalamus trinervis (Lam.) Hook. \& Arn., J. Bot. (Hooker) 3: 43. 1840. Pseudobaccharis trinervis (Lam.) V.M.Badillo, Bol. Soc. Venez. Ci. Nat. 10: 306. 1946. Psila trinervis (Lam.) Cabrera, Bol. Soc. Argent. Bot. 5: 211. 1955. Tipo: BRASIL. RIO DE JANEIRO: circa Sebastianopolis, VII.1767, @ e O", P. Commerson 
s.n. (holótipo P-Lam, O’; isótipos G-DC; P 594373 , foto!; P-218216, foto!).

Baccharis rhexioides Kunth in H.B.K., Nov. gen. sp. 4: 66. 1818. Molina rhexioides (Kunth) Less., Linnaea 6: 406. 1831. Baccharis trinervis var. rhexioides (Kunth) Bakerin Martius, Eichler \& Urban, Fl. bras. 6(3): 73. 1882. Pseudobaccharis rhexioides (Kunth) V.M.Badillo, Bol. Soc. Venez. Ci. Nat. 10: 306. 1946. Tipo: PERU: "juxta Montan, alt. 1400 hex.," \&, A. Humboldt \& A.J.A. Bonpland s.n. (holótipo P-HBK; isótipos B-W-15571-1; B15051, foto em F!; P-218218), syn. nov.

Baccharis divergens DC., Prodr. 5: 400. 1836. Tipo: MÉXICO: o, T.X.P. Haenke s.n. (holótipo GDC, foto!), syn. nov.

Baccharis oxyphylla DC., Prod. 5: 400. 1836. Tipo: PERU: o, E.F. Poeppig 24 (holótipo G-DC, foto!; isótipo P, n.v.), syn. nov.

Baccharis trichoclada DC., Prodr. 5: 400. 1836. Tipo: MÉXICO: o, T.X.P. Haenke s.n. (holótipo G-DC, foto!), syn. nov.

Baccharis eggersii Hieronymus, Bot. Jahrb. Syst. 28: 588. 1901. Tipo: ECUADOR: Guayas, Balao, I.1892, O", H.F.A. Eggers 14341 (lectótipo M, designado por Müller 2006, foto!; duplicatas L-2ex), syn. nov.

Barroso (1976) considerou B. rhexioides como uma variedade de $B$. trinervis. Contudo, a presente análise dos espécimes estudados por Barroso (1976) em RB demonstrou que os mesmos pertencem a $B$. bifrons Baker (Sucre 1163, 1936) e B. cinerea DC. (Brade 19389; Duarte 10535; Ferraz \& Lima s.n., RB77735; Vasconcelos 62; Vieira 34). Recentemente, Müller (2006) reconheceu a variedade B. trinervis var. rhexioides tendo citado dentre o material examinado espécimes coletados no Brasil.

Com base em fotografias dos tipos de Baccharis trinervis e $B$. rhexioides e em dois espécimes (Lowrie et al. 367; Forero et Wrigley 7072) citados por Müller (2006) como B. trinervis var. rhexioides, além do exame de uma ampla amostragem de espécimes coletados em território brasileiro, não foi possível reconhecer características morfológicas que permitissem a distinção desta variedade. Por essa razão e mediante a análise dos tipos, considera-se $B$. rhexioides e os binômios $B$. divergens, $B$. oxyphylla, B. trichoclada e B. eggersii, aceitos por Müller (2006) na sinonímia de $B$. trinervis var. rhexioides, como sinônimos de $B$. trinervis.

\section{Agradecimentos}

Os autores agradecem aos curadores dos herbários consultados o acesso às coleções e/ou empréstimo de material; ao Conselho Nacional de Desenvolvimento Científico e Tecnológico, a bolsa de mestrado CNPq-PROTAX concedida ao primeiro autor, e a Bolsa de Produtividade em Pesquisa, concedida ao segundo autor.

\section{Referências}

Baker, J.G. 1882. Compositae. III. Asteroideae, Inuloideae. In: Martius, C.F.P. von; Eichler, A.W. \& Urban, I. (ed.). Flora brasiliensis. München, Wien, Leipzig. Vol. 6, pars 3. Pp. 1-442.

Barroso, G.M. 1976. Compositae-Subtribo Baccharidinae Hoffman. Estudo das espécies ocorrentes no Brasil. Rodriguésia 28: 3-273.

Candolle, A.P. de 1836. Prodromus systematis naturalis regni vegetabilis 5 - Sistens Calycereas et Compositarum tribus priores. Treuttel \& Würtz, Paris.

Fielding, R.R. 2001. Baccharis: a genus of the Asteraceae new to Canada. Proceedings of the Nova Scotian Institute of Science 41: 214-215.

Giuliano, D.A. 2001. Classificación infragenérica de las espécies Argentinas de Baccharis (Asteraceae, Astereae). Darwiniana 39: 131-154.

Heiden, G. 2009. Estudos taxonômicos e conservação em Baccharis L. (Asteraceae; Astereae) no estado do Rio de Janeiro, Brasil. Dissertação de Mestrado. Escola Nacional de Botânica Tropical, Instituto de Pesquisas Jardim Botânico do Rio de Janeiro, Rio de Janeiro. 254p.

Malagarriga Heras, R.P. 1976 (1977). Nomenclator Baccharidinarum omnium. Memoria de la Sociedad de Ciencias Naturales La Salle 37: 129-224.

Müller, J. 2006. Systematics of Baccharis (CompositaeAstereae) in Bolivia, including an overview of the genus. Systematic Botany Monographs 76: 1-341.

Nesom, G.L. \& H. Robinson. 2006 (2007). XV. Tribe Astereae Cass. Vol. 8. In: Kadereit, J.W. \& C. Jeffrey (eds.), The families and genera of vascular plants (K. Kubitzki - series ed.). Flowering plants: Eudicots: Asterales. Springer, Berlin. Pp. 284-342.

Oliveira, A.S.; Deble, L.P.; Schneider, A.A. \& Marchiori, J.N.C. 2006. Checklist do gênero Baccharis L. para o Brasil (Asteraceae-Astereae). Balduinia 9: 17-27.

Oliveira-Deble, A.S. \& Deble, L.P. 2008. New species of Baccharis (Asteraceae, Astereae) from Brazil. Bonplandia 17: 13-24. 